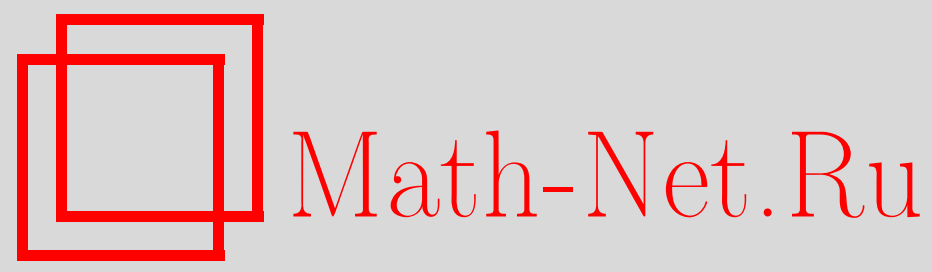

В. В. Басов, Бифуркация положения равновесия в системах с нулевыми корнями характеристического уравнения, Матем. заметки, 2004, том 75, выпуск 3, 323-341

DOI: https://doi.org/10.4213/mzm35

Использование Общероссийского математического портала Math-Net.Ru подразумевает, что вы прочитали и согласны с пользовательским соглашением http://www.mathnet.ru/rus/agreement

Параметры загрузки:

IP : 3.85 .73 .92

26 апреля 2023 г., 11:49:11 


\section{БИФУРКАЦИЯ ПОЛОЖЕНИЯ РАВНОВЕСИЯ \\ В СИСТЕМАХ С НУЛЕВЫМИ КОРНЯМИ \\ ХАРАКТЕРИСТИЧЕСКОГО УРАВНЕНИЯ}

\section{B. В. Басов}

Рассматривается вещественная автономная система $2 d$ дифференциальных уравнений с малым положительньм параметром $\varepsilon$ :

$$
\dot{x}_{i}=x_{i+d}+X_{i}^{(n+1)}(x, \varepsilon), \quad \dot{x}_{i+d}=-x_{i}^{2 n-1}+X_{i+d}^{(2 n)}(x, \varepsilon), \quad i=1, \ldots, d,
$$

где $d \geqslant 2, n \geqslant 2, X_{j}^{(k)}$ - непрерьвные и достаточное число раз непрерьвно дифференцируемые по $x, \varepsilon$ в окрестности нуля функции, разложение которых начинается с порядка $k$, если считать, что переменные $x_{i}$ имеют первый порядок малости, $\varepsilon$ - второй, а переменные $x_{i+d}$ - порядок $n$. Приводится конечное число явно выписанных условий на коэффициенты младших членов разложения правых частей этой системы, гарантирующих наличие у системы при любом достаточно малом $\varepsilon>0$ одного или нескольких $d$-мерных инвариантных торов с бесконечно малыми частотами движений на них.

Библиография: 8 названий.

1. Постановка задачи. Система (1). Рассмотрим вещественную автономную систему $2 d(d \geqslant 2)$ дифференциальных уравнений с мальм положительным параметром $\varepsilon$, матрица линейной части которой имеет $d$ пар нулевых собственных чисел с непростыми элементарными делителями,

$$
\begin{aligned}
\dot{x}_{i} & =x_{i+d}+X_{i}^{[n+1]}(x, \varepsilon)+X_{i}^{[n+2]}(x, \varepsilon)+X_{i}^{*[n+3]}(x, \varepsilon), \\
\dot{x}_{i+d} & =-x_{i}^{2 n-1}+X_{i+d}^{[2 n]}(x, \varepsilon)+X_{i+d}^{[2 n+1]}(x, \varepsilon)+X_{i+d}^{*[2 n+2]}(x, \varepsilon),
\end{aligned}
$$

где $i=1, \ldots, d, d \geqslant 2 ; x=\left(x^{\prime}, x^{\prime \prime}\right), x^{\prime}=\left(x_{1}, \ldots, x_{d}\right), x^{\prime \prime}=\left(x_{d+1}, \ldots, x_{2 d}\right) ; n \geqslant 2$; $X_{\iota}^{[k]}(x, \varepsilon)$ - однородные полиномы $k$-й степени, $\iota=1, \ldots, 2 d$, если считать, что переменные $x_{i}$ имеют первьй порядок малости, $\varepsilon-$ второй, $x_{i+d}$-порядок $n$; функции $X_{\iota}^{*[k]}$ непрерьвны на множестве $H=\left\{(x, \varepsilon):\|x\|<x_{0}, 0 \leqslant \varepsilon<\varepsilon_{0}\right\}, X_{\iota}^{*[k]}\left(\varepsilon^{1 / 2} x^{\prime}, \varepsilon^{n / 2} x^{\prime \prime}, \varepsilon\right)=$ $\varepsilon^{k} \widehat{X}_{\iota}^{*[k]}\left(x^{\prime}, x^{\prime \prime}, \varepsilon\right), \widehat{X}_{\iota}^{*[k]}$ - достаточное число раз непрерьвно дифференцируемые в $H$ функции.

Работа выполнена при поддержке Российского фонда фундаментальных исследований, грант № 02-01-00245. 
Выбор порядков для переменных $x_{i}, x_{i+d}$ и $\varepsilon$ обусловлен необходимостью осуществления обобщенной полярной замены Ляпунова (5) и стандартной аффинной замены (7), приведенных в п. 2.

Таким образом, рассматривается ситуация, когда в уравнениях для $\dot{x}^{\prime \prime}$ системы (1) младшие члены имеют отрицательные знаки и одинаковые нечетные степени, а значит, для нулевого приближения каждой пары уравнений с номерами $i$ и $i+d$ реализуется случай центра.

Полиномы $X_{\iota}^{[k]}(x, \varepsilon)$ удобно записьвать в следующем виде:

$$
X_{\iota}^{[2 m+\nu]}=\sum_{\mu=0}^{m} X_{\iota 2 m+\nu}^{[2 \mu+\nu]}(x) \varepsilon^{m-\mu}, \quad X_{\iota k}^{[\varkappa]}=\sum_{\left|p^{\prime}\right|+n\left|p^{\prime \prime}\right|=\varkappa} X_{\iota k}^{\left(p^{\prime}, p^{\prime \prime}\right)} x^{p}
$$

где $m \geq 1, \nu=0,1, \iota=1, \ldots, 2 d, p=\left(p_{1}, \ldots, p_{2 d}\right)$ имеет целые неотрищательные компоненты, $p^{\prime}=\left(p_{1}, \ldots, p_{d}\right),|p|=p_{1}+\cdots+p_{2 d}, x^{p}=x_{1}^{p_{1}} \ldots x_{2 d}^{p_{2 d}}$.

Целью настоящей работы является получение явных условий на коэффициенты полиномов $X_{\iota}^{[k]}$, гарантирующих при всех достаточно малых положительных значениях $\varepsilon$ рождение у системы (1) $d$-мерных инвариантных торов, но говорить о бифуркации положения равновесия системы (1) можно только в том случае, когда $X_{\iota}^{[k]}(0, \varepsilon) \equiv 0$ и $X_{\iota}^{*[k]}(0, \varepsilon) \equiv 0$.

В работе $[1$, гл. $1, \S 1]$ поставленная задача решена для случая $n=1$, когда матрица линейной части системы (1) имеет $d$ пар чисто мнимых собственных чисел. Там же описана процедура нахождения инвариантных торов размерности большей, чем $d$.

Как будет видно из дальнейших рассуждений, решающую роль при доказательстве существования инвариантных торов у системы (1) при $n \geqslant 2$ играет наличие положительных решений в алгебраической бифуркационной системе уравнений $V(\alpha)=0$, вектор-функция $V(\alpha)$ которой при четных $n$ задается формулой $\left(14_{\mathrm{ev}}\right)$ из п. 4 и содержит только коэффициенты полиномов $X_{i}^{[n+1]}$ и $X_{i+d}^{[2 n]}$ системы (1), а при нечетных $n$ - формулой $(\breve{12}$ оd $)$ из п. 7. Все ограничения на решения бифуркационной системы, а их конечное число, в явном виде представлены в условиях (18) и (ૅ ной в п. 9, их достаточно для существования у системы (1) при любом достаточно малом значении параметра $\varepsilon>0$ одного или нескольких инвариантных торов размерности $d \mathrm{c}$ бесконечно мальми по отношению к амплитудам частотами движений на них.

Следует отметить важное отличие систем с чисто мнимьми характеристическими корнями из [1], где $n=1$, от систем, рассматриваемых в настоящей работе, где $n \geqslant 2$ и, следовательно, все корни равны нулю. При $n=1$ для реализации процесса осреднения на собственные числа матрицы линейной части исходной системы накладьвается условие Зигеля на "малые знаменатели”. Аналогичное условие непосредственно в системе (1) при $n \geqslant 2$ накладьвать не на что. Оказывается, ему должны будут удовлетворять корни бифуркационной системы уравнений - условие $\left(18_{2}\right)$, не имевшие при $n=1$ подобных ограничений.

В п. 10 и п. 11 рассматриваются два характерных и весьма интересных частных случая для системы (1), в частности, когда она вообще не зависит от малого параметра, что позволяет, наложив пару условий на коэффициенты, найти у нее бесконечные семейства инвариантных торов. 
Отметим также, что полученные ниже результаты имеют непосредственноеприкладное значение, так как частньм случаем системы (1) является система $d$ дифференциальных уравнений второго порядка с малым положительным параметром $\varepsilon$ :

$$
\ddot{y}+y^{2 n-1}=Y(y, \dot{y}, \varepsilon),
$$

описьвающая колебания $d$ слабосвязанных осцилляторов с восстанавливающими силами порядка $2 n-1$. В (3) $y=\left(y_{1}, \ldots, y_{d}\right), y^{k}=\left(y_{1}^{k}, \ldots, y_{d}^{k}\right)$.

Особенность системы (3) заключается в том, что при $n \geqslant 2$ невозмущенные колебания каждого из осцилляторов имеют частоты, которые бесконечно малы при стремлении к нулю амплитуд колебаний.

Действительно, замена $y=x^{\prime}, \dot{y}_{1}=x^{\prime \prime}$ преобразует (3) в систему

$$
\dot{x}^{\prime}=x^{\prime \prime}, \quad \dot{x}^{\prime \prime}=-x^{2 n-1}+Y\left(x^{\prime}, x^{\prime \prime}, \varepsilon\right) .
$$

Пусть в системе (4) $Y_{i}=X_{i+d}^{[2 n]}(x, \varepsilon)+X_{i+d}^{[2 n+1]}(x, \varepsilon)+X_{i+d}^{*[2 n+2]}(x, \varepsilon)$, где $i=1, \ldots, d$, $X_{i+d}^{[k]}, X_{i+d}^{*[k]}$ описаны в системе $(1)$. Тогда для системы (3) с указанными правыми частями справедливы все результаты, которые получены ниже для системы (1).

2. Полярная и аффинная замены. Система (8). Сделаем в системе (1) обобщенную полярную замену переменных, предложенную А. М. Ляпуновым в [2, с. 290]:

$$
x_{i}=r_{i} \operatorname{Cs} \varphi_{i}, \quad x_{i+d}=-r_{i}^{n} \operatorname{Sn} \varphi_{i}, \quad i=1, \ldots, d,
$$

или в векторной записи: $x^{\prime}=r \operatorname{Cs} \varphi, x^{\prime \prime}=-r^{n} \operatorname{Sn} \varphi$, в которой функции $\operatorname{Cs} \varphi_{i}, \operatorname{Sn} \varphi_{i}$ определяются уравнениями $\operatorname{Cs}^{\prime} \varphi_{i}=-\operatorname{Sn} \varphi_{i}, \operatorname{Sn}^{\prime} \varphi_{i}=\operatorname{Cs}^{2 n-1} \varphi_{i}$ и начальными данными $\operatorname{Cs} 0=1$, Sn $0=0$. При таком определении Cs - четная, a Sn - нечетная периодические функции с периодом $\omega$, зависящие от параметра $n$, и $\operatorname{Cs}^{2 n} \varphi_{i}+n \operatorname{Sn}^{2} \varphi_{i}=1$.

В результате полярной замены (5) из системы (1) получаем систему

$$
\begin{aligned}
r_{i}^{n-1} \dot{r}_{i} & =r_{i}^{n-1} \operatorname{Cs}^{2 n-1} \varphi_{i}\left(X_{i}^{[n+1]}+\cdots\right)-\operatorname{Sn} \varphi_{i}\left(X_{i+d}^{[2 n]}+\cdots\right), \\
r_{i}^{n} \dot{\varphi}_{i} & =r_{i}^{2 n-1}-n r_{i}^{n-1} \operatorname{Sn} \varphi_{i}\left(X_{i}^{[n+1]}+\cdots\right)-\operatorname{Cs} \varphi_{i}\left(X_{i+d}^{[2 n]}+\cdots\right)
\end{aligned}
$$

или, что то же самое, систему

$$
\begin{aligned}
& \dot{r}_{i}=R_{i}^{[1]}(r, \varphi, \varepsilon)+R_{i}^{[2]}(r, \varphi, \varepsilon)+R_{i}^{*[3]}(r, \varphi, \varepsilon), \quad i=1, \ldots, d, \\
& \dot{\varphi}_{i}=r_{i}^{n-1}+r_{i}^{-1}\left(\Phi_{i}^{[1]}(r, \varphi, \varepsilon)+\Phi_{i}^{*[2]}(r, \varphi, \varepsilon)\right),
\end{aligned}
$$

в которой

$$
\begin{aligned}
& R_{i}^{[1]}(r, \varphi, \varepsilon)=\operatorname{Cs}^{2 n-1} \varphi_{i} X_{i}^{[n+1]}\left(r \operatorname{Cs} \varphi,-r^{n} \operatorname{Sn} \varphi, \varepsilon\right)-r_{i}^{1-n} \operatorname{Sn} \varphi_{i} X_{i+d}^{[2 n]}(\ldots), \\
& R_{i}^{[2]}(r, \varphi, \varepsilon)=\operatorname{Cs}^{2 n-1} \varphi_{i} X_{i}^{[n+2]}\left(r \operatorname{Cs} \varphi,-r^{n} \operatorname{Sn} \varphi, \varepsilon\right)-r_{i}^{1-n} \operatorname{Sn} \varphi_{i} X_{i+d}^{[2 n+1]}(\ldots), \\
& \Phi_{i}^{[1]}(r, \varphi, \varepsilon)=-n \operatorname{Sn} \varphi_{i} X_{i}^{[n+1]}\left(r \operatorname{Cs} \varphi,-r^{n} \operatorname{Sn} \varphi, \varepsilon\right)-r_{i}^{1-n} \operatorname{Cs} \varphi_{i} X_{i+d}^{[2 n]}(\ldots),
\end{aligned}
$$

т.е. многочлены $R_{i}^{[\varkappa]}, \Phi_{i}^{[\varkappa]}$ имеют суммарный порядок $n+\varkappa$ по $r_{j}$ и $\varepsilon^{1 / 2}$, а функции $R_{i}^{*[3]}$, $\Phi_{i}^{*[2]}$ являются непрерывными и гладкими функциями $r_{j}, \varepsilon, \omega$-периодическими по $\varphi_{j}$ и начинаются по $r_{j}, \varepsilon^{1 / 2}$ не ниже чем с порядков $n+3$ и $n+2$ соответственно, $j=1, \ldots, d$. 
Сделаем в системе (6) аффинную замену переменных

$$
r_{i}=\varepsilon^{1 / 2}\left(\alpha_{i}+u_{i}\right), \quad i=1, \ldots, d,
$$

где $\alpha=\left(\alpha_{1}, \ldots, \alpha_{d}\right)$ - постоянный вектор, $\alpha_{i}>0,\left|u_{i}\right|<\alpha_{i}$.

В результате аффинной замены (7) из (6) получаем систему

$$
\begin{aligned}
& \varepsilon^{1 / 2} \dot{u}_{i}=R_{i}^{[1]}\left(\varepsilon^{1 / 2}(\alpha+u), \varphi, \varepsilon\right)+R_{i}^{[2]}(\ldots)+R_{i}^{*[3]}(\ldots), \\
& \dot{\varphi}_{i}=\varepsilon^{n / 2-1 / 2}\left(\alpha_{i}+u_{i}\right)^{n-1}+\varepsilon^{-1 / 2}\left(\alpha_{i}+u_{i}\right)^{-1}\left(\Phi_{i}^{[1]}(\ldots)+\Phi_{i}^{*[2]}(\ldots)\right)
\end{aligned}
$$

или, учитьвая структуру $R$ и $\Phi$, систему

$$
\begin{aligned}
\dot{u} & =\varepsilon_{n}\left(\varepsilon^{1 / 2} R_{i}^{[1]}(\alpha+u, \varphi, 1)+\varepsilon R_{i}^{[2]}(\alpha+u, \varphi, 1)+O\left(\varepsilon^{3 / 2}\right)\right), \\
\dot{\varphi}_{i} & =\varepsilon_{n}\left(\left(\alpha_{i}+u_{i}\right)^{n-1}+\varepsilon^{1 / 2}\left(\alpha_{i}+u_{i}\right)^{-1} \Phi_{i}^{[1]}(\alpha+u, \varphi, 1)+O(\varepsilon)\right),
\end{aligned}
$$

в которой $\varepsilon_{n}=\varepsilon^{n / 2-1 / 2}$.

3. Первичное осреднение системы (8). Система (10). В дальнейшем любые функции, зависящие от $\varphi=\left(\varphi_{1}, \ldots, \varphi_{d}\right)$, будут $\omega$-периодическими функциями $\varphi_{i}$. Введем разложение

$$
\Upsilon(\varphi)=\bar{\Upsilon}+\widetilde{\Upsilon}(\varphi), \quad \bar{\Upsilon}=\omega^{-d} \int_{0}^{\omega} \ldots \int_{0}^{\omega} \Upsilon(\varphi) d \varphi_{1} \ldots d \varphi_{d}-\text { среднее } \Upsilon .
$$

Тогда $\int \widetilde{\Upsilon}(\varphi) d \varphi$, имея подьнтегральную функцию с нулевым средним, будет периодической функцией, которая выбором нижних пределов интегрирования однозначно определяется из условия $\int_{\varphi_{0}}^{\varphi} \widetilde{\Upsilon}(\varphi) d \varphi=\widetilde{v}(\varphi)$, т.е. имеет нулевое среднее значение. Обозначаем $\Upsilon^{(j)}(\alpha, \varphi)=\partial \Upsilon(\alpha, \varphi) / \partial \alpha_{j}, \Upsilon^{(j k)}(\alpha, \varphi)=\partial^{2} \Upsilon(\alpha, \varphi) /\left(\partial \alpha_{j} \partial \alpha_{k}\right)$.

Возвращаясь к системе (8), покажем, что существует замена

$$
u_{i}=v_{i}+\varepsilon^{1 / 2}\left(\tilde{f}_{i}(\alpha, \varphi)+\sum_{j=1}^{d} \tilde{f}_{i}^{(j)}(\alpha, \varphi) v_{j}+\frac{1}{2} \sum_{j, k=1}^{d} \tilde{f}_{i}^{(j k)}(\alpha, \varphi) v_{j} v_{k}\right)
$$

переводящая (8) в систему

$$
\left\{\begin{aligned}
\dot{v}_{i}= & \varepsilon_{n}\left(\varepsilon^{1 / 2}\left(V_{i}(\alpha)+\sum_{j=1}^{d} V_{i}^{(j)}(\alpha) v_{j}+\frac{1}{2} \sum_{j, k=1}^{d} V_{i}^{(j k)}(\alpha) v_{j} v_{k}\right)\right. \\
& \left.+\varepsilon\left(W_{i}(\alpha, \varphi)+\sum_{j=1}^{d} W_{i}^{(j)}(\alpha, \varphi) v_{j}\right)+O\left(\varepsilon^{1 / 2}|v|^{3}+\varepsilon|v|^{2}+\varepsilon^{3 / 2}\right)\right) \\
\dot{\varphi}_{i}= & \varepsilon_{n}\left(\left(\alpha_{i}+v_{i}\right)^{n-1}+\varepsilon^{1 / 2}\left(\Phi_{i}(\alpha, \varphi)+\sum_{j=1}^{d} \Phi_{i}^{(j)}(\alpha, \varphi) v_{j}\right)\right. \\
& \left.+O\left(\varepsilon^{1 / 2}|v|^{2}+\varepsilon\right)\right)
\end{aligned}\right.
$$


Продифференцируем замену (9) $u=v+\varepsilon^{1 / 2} \tilde{f}(v, \alpha, \varphi)$ в силу систем (8) и (10) и сократим полученные равенства на $\varepsilon_{n}$; тогда

$$
\begin{aligned}
\varepsilon^{1 / 2} R_{i}^{[1]}(\alpha & \left.+v+\varepsilon^{1 / 2} \tilde{f}, \varphi, 1\right)+\varepsilon R_{i}^{[2]}\left(\alpha+v+\varepsilon^{1 / 2} \tilde{f}, \varphi, 1\right)+O\left(\varepsilon^{3 / 2}\right) \\
= & \varepsilon^{1 / 2}\left(V_{i}+\sum_{j=1}^{d} V_{i}^{(j)} v_{j}+\frac{1}{2} \sum_{j, k=1}^{d} V_{i}^{(j k)} v_{j} v_{k}\right) \\
& +\varepsilon\left(W_{i}+\sum_{j=1}^{d} W_{i}^{(j)} v_{j}\right)+O\left(\varepsilon^{1 / 2}|v|^{3}+\varepsilon|v|^{2}+\varepsilon^{3 / 2}\right) \\
& +\varepsilon^{1 / 2}\left(\sum_{j=1}^{d} \tilde{f}_{i}^{(j)}+\sum_{j, k=1}^{d} \tilde{f}_{i}^{(j k)} v_{k}\right)\left(\varepsilon^{1 / 2}\left(V_{j}+\sum_{k=1}^{d} V_{j}^{(k)} v_{k}\right)+O\left(\varepsilon^{1 / 2}|v|^{2}+\varepsilon\right)\right) \\
& +\varepsilon^{1 / 2} \sum_{\nu=1}^{d}\left(\frac{\partial \tilde{f}_{i}}{\partial \varphi_{\nu}}+\sum_{j=1}^{d} v_{j} \frac{\partial \tilde{f}_{i}^{(j)}}{\partial \varphi_{\nu}}+\frac{1}{2} \sum_{j, k=1}^{d} v_{j} v_{k} \frac{\partial \tilde{f}_{i}^{(j k)}}{\partial \varphi_{\nu}}\right) \\
& \times\left(\left(\alpha_{\nu}+v_{\nu}\right)^{n-1}+\varepsilon^{1 / 2} \Phi_{\nu}+\varepsilon^{1 / 2} \sum_{j=1}^{d} \Phi_{\nu}^{(j)} v_{j}+O\left(\varepsilon^{1 / 2}|v|^{2}+\varepsilon\right)\right) .
\end{aligned}
$$

Приравнивая в (11) слагаемые, стоящие при $\varepsilon^{1 / 2}$, получаем

$$
\sum_{\nu=1}^{d} \frac{\partial \tilde{f}_{i}(\alpha, \varphi)}{\partial \varphi_{\nu}} \alpha_{\nu}^{n-1}=R_{i}^{[1]}(\alpha, \varphi, 1)-V_{i}(\alpha), \quad i=1, \ldots, d
$$

Потребуем, чтобы постоянньй вектор $\alpha$ с положительными компонентами из аффинной замены (7) удовлетворял условию

$$
\begin{aligned}
\exists \gamma & >0, \quad \tau \geqslant d: \forall q=\left(q_{1}, \ldots, q_{d}\right) \neq 0, \quad q_{i} \text { - целье } \\
& \Rightarrow\left|\alpha_{1}^{n-1} q_{1}+\cdots+\alpha_{d}^{n-1} q_{d}\right|>\gamma\left(\left|q_{1}\right|+\cdots+\left|q_{d}\right|\right)^{-\tau}
\end{aligned}
$$

Условие (13) означает, что $\alpha_{1}, \ldots, \alpha_{d}$ рационально несоизмеримы и вектор $\alpha$ удовлетворяет условию Зигеля на "малые знаменатели".

Положим

$$
V_{i}(\alpha)=\overline{R_{i}^{[1]}(\alpha, \varphi, 1)}=\omega^{-d} \int_{0}^{\omega} \cdots \int_{0}^{\omega} R_{i}^{[1]}(\alpha, \varphi, 1) d \varphi_{1} \ldots d \varphi_{d}
$$

Тогда правые части (12), являясь полиномами относительно $\operatorname{Cs} \varphi_{j}$ и $\operatorname{Sn} \varphi_{j}$, представляют собой вещественно-аналитические $\omega$-периодические с нулевыми средними функции $\varphi_{1}, \ldots, \varphi_{d}$, сходящиеся в некоторой окрестности вещественной оси, поскольку таковыми являются $\operatorname{Cs} \varphi_{j}$ и $\operatorname{Sn} \varphi_{j}$.

В работе [1, с. 16] доказано следующее утверждение. 
Лемма В4. Рассмотрим уравнение $\sum_{\nu=1}^{d}\left(\partial \tilde{f}(\varphi) / \partial \varphi_{\nu}\right) \alpha_{\nu}=\widetilde{F}(\varphi)$, әде $F$ есть $2 \pi$-периодическая по всем своим аргументам, аналитическая и ограниченная при $|\operatorname{Im} \varphi|<\delta$ скалярная функиия с нулевым средним, удовлетворяющая (13) с $n=2$. Тогда для любого $\rho, 0<\rho<\delta$, существует $2 \pi$-периодическое аналитическое при $|\operatorname{Im} \varphi|<\rho$ решение уравнения $\tilde{f}(\varphi)$, к которого $\sup _{|\operatorname{Im} \varphi|<\rho}|f|<c(d, \tau, \gamma) \times$ $(\delta-\rho)^{-\tau-1} \sup _{|\operatorname{Im} \varphi|<\delta}|F|$.

Согласно этой лемме уравнения (12) при условиях (13) и выбранных в (14) средних значениях $V_{i}(\alpha)$ однозначно разрешимы, причем их решения $\tilde{f}_{i}(\alpha, \varphi)$ обладают теми же свойствами, что и правые части (12).

Если теперь в (11) приравнять слагаемые, стоящие при $\varepsilon^{1 / 2} v_{j}$, то для любых $i, j=$ $1, \ldots, d$ получим равенство

$$
\sum_{\nu=1}^{d} \frac{\partial \tilde{f}_{i}^{(j)}(\alpha, \varphi)}{\partial \varphi_{\nu}} \alpha_{\nu}^{n-1}=R_{i}^{[1](j)}(\alpha, \varphi, 1)-V_{i}^{(j)}(\alpha)-\frac{\partial \tilde{f}_{i}(\alpha, \varphi)}{\partial \varphi_{j}}(n-1) \alpha_{j}^{n-2}
$$

котороевсегда вьполняется, так как может быть получено дифференцированием (12) по $\alpha_{j}$. Очевидно, что $V_{i}^{(j)}(\alpha)=\overline{R_{i}^{[1](j)}(\alpha, \varphi, 1)}$, поскольку последнее слагаемое последнего равенства имеет нулевое среднее значение.

Аналогичным образом равенство, получаемое приравниванием слагаемых при $\varepsilon^{1 / 2} v_{j} v_{k}$, совпадает со второй производной (12) по $\alpha_{j} \alpha_{k}$.

В свою очередь, непосредственная подстановка замены (9) в уравнения для $\dot{\varphi}_{i}$ системы (8) и выделение членов, стоящих при $\varepsilon^{1 / 2}$, дает

$$
\Phi_{i}(\alpha, \varphi)=(n-1) \alpha_{i}^{n-2} \tilde{f}_{i}+\alpha_{i}^{-1} \Phi_{i}^{[1]}(\alpha, \varphi, 1),
$$

а выделение членов, стоящих при $\varepsilon^{1 / 2} v_{j}$, дает такое же равенство, какое можно получить после дифференцирования (15) по $\alpha_{j}$.

И, наконец, приравнивая в (11) слагаемые, стоящие при $\varepsilon$ и при $\varepsilon v_{j}$, находим функции $W_{i}$ и $W_{i}^{(j)}$ :

$$
W_{i}(\alpha, \varphi)=R_{i}^{[2]}(\alpha, \varphi, 1)+\sum_{j=1}^{d} R_{i}^{[1](j)}(\alpha, \varphi, 1) \tilde{f}_{j}-\sum_{j=1}^{d} \tilde{f}_{i}^{(j)} V_{j}-\sum_{\nu=1}^{d} \frac{\partial \tilde{f}_{i}}{\partial \varphi_{\nu}} \Phi_{\nu}
$$

a $W_{i}^{(j)}(\alpha, \varphi)$ получается после дифференцирования (16) по $\alpha_{j}$.

Таким образом, первичная осредняющая замена (9) построена. При условии (13) она преобразует (8) в систему $(10)$, в которой $V_{i}(\alpha)$ вычисляются по формулам (14) и зависят только от $R_{i}^{[1]}(\alpha, \varphi, 1)$ из $(6)$.

4. Бифуркационная система для четных $n$. Система $\left(10_{\mathrm{ev}}^{*}\right)$. Функции $V_{i}(\alpha)$ играют решающую роль при нахождении у системы (1) $d$-мерных инвариантных торов. Ниже будет показано, что величина $V=\left(V_{1}, \ldots, V_{d}\right)$ существенным образом зависит от четности числа $n$.

Пусть $n=2 m, m \geqslant 1$. Тогда в (6)

$$
R_{i}^{[1]}(\alpha, \varphi, 1)=\mathrm{Cs}^{4 m-1} \varphi_{i} \sum_{\mu=0}^{m} X_{i 2 m+1}^{[2 \mu+1]}-\alpha_{i}^{1-2 m} \operatorname{Sn} \varphi_{i} \sum_{\mu=0}^{2 m} X_{i+d 4 m}^{[2 \mu]},
$$


где функции $X_{\iota k}^{[\varkappa]}$, определенные формулами (2), имеют согласно (5) аргументы $\alpha \operatorname{Cs} \varphi$, $-\alpha^{2 m} \operatorname{Sn} \varphi$ и порядок $\varkappa$ по $\alpha_{1}, \ldots, \alpha_{d}$.

Очевидно, что любое слагаемое в (17), содержащее хотя бы один $\operatorname{Sn} \varphi_{j}$ или $\operatorname{Cs} \varphi_{j}$ в нечетной степени, имеет нулевое среднее значение и не влияет на величину $V(\alpha)$ в $(14)$. Поэтому выделим из правой части (17) те слагаемые, которые содержат только четные степени обобщенных синусов и косинусов, обозначив их $R_{i, \mathrm{ev}}^{[1]}(\alpha, \varphi)$. При $n=2 m$ имеем

$$
R_{i, \mathrm{ev}}^{[1]}=\alpha_{i} \sum_{\mu=0}^{m} \sum_{l:|l|=\mu}\left(\mathrm{Cs}^{4 m} \varphi_{i} X_{i 2 m+1}^{\left(2 l+e_{i}, 0\right)}+\operatorname{Sn}^{2} \varphi_{i} X_{i+d 4 m}^{\left(2 l, e_{i}\right)}\right) \prod_{j=1}^{d} \alpha_{j}^{2 l_{j}} \mathrm{Cs}^{2 l_{j}} \varphi_{j}
$$

Учитывая, что $\overline{R_{i}^{[1]}}=\overline{R_{i, \text { ev }}^{[1]}}$, а также формулы

$$
\mathrm{Cs}^{4 m} \varphi_{i}+2 m \operatorname{Sn}^{2} \varphi_{i}=1, \quad \int_{0}^{\omega} \operatorname{Cs}^{2 n+k} \varphi_{i} d \varphi_{i}=\frac{k+1}{n+k+1} \int_{0}^{\omega} \operatorname{Cs}^{k} \varphi_{i} d \varphi_{i}
$$

при помощи (14) найдем $V_{i}$ для четных $n$ :

$$
V_{i}(\alpha)=\alpha_{i} \sum_{l:|l|=0}^{m} \frac{\varkappa_{n}^{(l)}}{n+2 l_{i}+1}\left(\left(2 l_{i}+1\right) X_{i 2 m+1}^{\left(2 l+e_{i}, 0\right)}+X_{i+d 4 m}^{\left(2 l, e_{i}\right)}\right) \alpha^{2 l},
$$

где $i=1, \ldots, d, l=\left(l_{1}, \ldots, l_{d}\right), l_{i} \geqslant 0, \varkappa_{n}^{(l)}=\frac{1}{\omega^{d}} \prod_{j=1}^{d} \int_{0}^{\omega} \operatorname{Cs}^{2 l_{j}} \varphi_{j} d \varphi_{j}, \alpha^{2 l}=$ $\left(\alpha_{1}^{2 l_{1}}, \ldots, \alpha_{d}^{2 l_{d}}\right), e_{i}=\left(0, \ldots, 1_{i}, \ldots, 0\right), n=2 m$.

ОПРедЕлЕниЕ 1. Алгебраическая система $V(\alpha)=0$, где полином $V$ задан формулой $\left(14_{\text {ev }}\right)$, называется бифуркационной системой уравнений для системы (1), в которой $n=2 m$.

ЗАмЕчАнИЕ 1 . При $n=2 m$ бифуркационная система - это алгебраическая система $d$ уравнений степени $m$ относительно $\alpha_{1}^{2}, \ldots, \alpha_{d}^{2}$. В нее входят только коэффициенты полиномов $X_{i}^{[n+1]}$ и $X_{i+d}^{[2 n]}$ системы $(1)$.

Введем матрицу $V_{L}(\alpha)=\left\{V_{i}^{(j)}(\alpha)\right\}_{i, j=1}^{d}$, где $V_{i}^{(j)}=\partial V_{i} / \partial \alpha_{j}$. Предположим, что $\exists \alpha^{*}=\left(\alpha_{1}^{*}, \ldots, \alpha_{d}^{*}\right)>0$ :

1) $V\left(\alpha^{*}\right)=0$

2) $\alpha^{*}$ удовлетворяет условию (13);

3) $V_{L}\left(\alpha^{*}\right)$ - некритическая матрища.

Используя $\left(14_{\text {ev }}\right)$ и $\left(18_{1}\right)$, подсчитаем матрицу $V_{L}\left(\alpha^{*}\right)$ :

$$
V_{i}^{(j)}\left(\alpha^{*}\right)=\sum_{l:|l|=1}^{m} \frac{2 l_{j} \varkappa_{n}^{(l)}}{n+2 l_{i}+1}\left(\left(2 l_{i}+1\right) X_{i 2 m+1}^{\left(2 l+e_{i}, 0\right)}+X_{i+d 4 m}^{\left(2 l, e_{i}\right)}\right)\left(\alpha^{*}\right)^{2 l+e_{i}-e_{j}} .
$$

Условия (18) окажется достаточно для доказательства существования у системы (1) при любых малых $\varepsilon>0 d$-мерных инвариантных торов.

Подставим вектор $\alpha=\alpha^{*}$, удовлетворяющий (18), в замены (7), (9) и системы (8), (10); тогда условие $\left(18_{2}\right)$ обеспечит существование замены $(9)$, условие $\left(18_{1}\right)$ аннулирует в радиальных уравнениях системы (10) члены, стоящие при $\varepsilon_{n} \varepsilon$, а условие $\left(18_{1}\right)$ 
гарантирует, что матрица линейной части членов, стоящих при той же степени $\varepsilon$, не имеет собственньх чисел с нулевой вещественной частью.

В результате система (10) с $n=2 m$ и $\alpha=\alpha^{*}$ из (18) принимает вид

$$
\begin{aligned}
\dot{v} & =\varepsilon_{n}\left(\varepsilon^{1 / 2} V_{L}\left(\alpha^{*}\right) v+\varepsilon W\left(\alpha^{*}, \varphi\right)+O\left(\varepsilon^{1 / 2}|v|^{3}+\varepsilon|v|+\varepsilon^{3 / 2}\right)\right), \\
\dot{\varphi}_{i} & =\varepsilon_{n}\left(\left(\alpha_{i}^{*}+v_{i}\right)^{2 m-1}+\varepsilon^{1 / 2} \Phi_{i}\left(\alpha^{*}, \varphi\right)+O\left(\varepsilon^{1 / 2}|v|+\varepsilon\right)\right) .
\end{aligned}
$$

ЗАмЕчАНИЕ 2 . При $n=2 m$ в системе (8) для последующих целей было бы достаточно осреднить только свободные и линейные по $v$ члены, стоящие при $\varepsilon_{n} \varepsilon^{1 / 2}$, при помощи замены переменных

$$
u=v+\varepsilon^{1 / 2}\left(\tilde{f}(\alpha, \varphi)+\tilde{f}_{L}(\alpha, \varphi) v\right),
$$

где матрица $\tilde{f}_{L}=\left\{\tilde{f}_{i}^{(j)}(\alpha, \varphi)\right\}_{i, j=1}^{d}$. Тогдав первом уравнении $\left(10_{\mathrm{ev}}^{*}\right)$ вместо $O\left(\varepsilon^{1 / 2}|v|^{3}+\right.$ $\cdots)$ стояло бы $O\left(\varepsilon^{1 / 2}|v|^{2}+\cdots\right)$.

5. Процесс осреднения системы $\left(10_{\mathrm{ev}}^{*}\right)$. Система (25). Для того чтобы можно было применить лемму Хейла о существовании при всех достаточно малых положительных значениях $\varepsilon d$-мерного инвариантного $\omega$-периодического многообразия, в системе $\left(10_{\mathrm{ev}}^{*}\right)$ надо предварительно осреднить члены, стоящие при младших степенях $\varepsilon$ и зависящие только от $\varphi$ как в уравнениях для радиальных, так и в уравнениях для угловых переменных. Благодаря условию $(183)$ сделать это всегда возможно, поскольку матрица $V_{L}\left(\alpha^{*}\right)$ имеет обратную.

Покажем сначала, что замена

$$
v=w-\varepsilon^{1 / 2} \bar{g}\left(\alpha^{*}\right)+\varepsilon \tilde{g}\left(\alpha^{*}, \varphi\right)
$$

аннулирует в $\left(10_{\mathrm{ev}}^{*}\right)$ слагаемое $\varepsilon W\left(\alpha^{*}, \varphi\right)$, преобразуя $\left(10_{\mathrm{ev}}^{*}\right)$ в систему

$$
\begin{aligned}
\dot{w} & =\varepsilon_{n}\left(\varepsilon^{1 / 2} V_{L}\left(\alpha^{*}\right) w+O\left(\varepsilon^{1 / 2}|w|^{3}+\varepsilon|w|+\varepsilon^{3 / 2}\right)\right), \\
\dot{\varphi}_{i} & =\varepsilon_{n}\left(\left(\alpha_{i}^{*}+w_{i}\right)^{2 m-1}+\varepsilon^{1 / 2} \Phi_{i}^{0}\left(\alpha^{*}, \varphi\right)+O\left(\varepsilon^{1 / 2}|w|+\varepsilon\right)\right),
\end{aligned}
$$

где $\Phi_{i}^{0}\left(\alpha^{*}, \varphi\right)=\Phi_{i}\left(\alpha^{*}, \varphi\right)-(2 m-1) \alpha_{i}^{* 2 m-2} V_{L}^{-1}\left(\alpha^{*}\right) \bar{W}\left(\alpha^{*}\right), w=\left(w_{1}, \ldots, w_{n}\right)$.

Дифференцируя замену (20) в силу систем $\left(10_{\mathrm{ev}}^{*}\right)$ и $(21)$, получаем

$$
\begin{array}{r}
\varepsilon^{1 / 2} V_{L}\left(w-\varepsilon^{1 / 2} \bar{g}+\varepsilon \tilde{g}\right)+\varepsilon W+O\left(\varepsilon^{1 / 2}|w|^{3}+\varepsilon|w|+\varepsilon^{3 / 2}\right) \\
=\varepsilon^{1 / 2} V_{L} w+\varepsilon \sum_{\nu=1}^{d} \frac{\partial \tilde{g}}{\partial \varphi_{\nu}}\left(\left(\alpha_{\nu}^{*}+w_{\nu}\right)^{2 m-1}+O\left(\varepsilon^{1 / 2}\right)\right),
\end{array}
$$

откуда $\sum_{\nu=1}^{d} \alpha_{\nu}^{* 2 m-1} \partial \tilde{g} / \partial \varphi_{\nu}=W\left(\alpha^{*}, \varphi\right)-V_{L}\left(\alpha^{*}\right) \bar{g}\left(\alpha^{*}\right)$. Это уравнение однозначно разрешимо благодаря условиям (18) при $\bar{g}=V_{L}^{-1}\left(\alpha^{*}\right) \bar{W}\left(\alpha^{*}\right)$.

Покажем теперь, что существует замена угловых переменных

$$
\varphi_{i}=\psi_{i}+\varepsilon^{1 / 2} \widetilde{\theta}_{i}(\psi), \quad \psi=\left(\psi_{1}, \ldots, \psi_{d}\right), \quad i=1, \ldots, d,
$$

которая преобразует систему (21) в систему

$$
\begin{aligned}
\dot{w} & =\varepsilon_{n}\left(\varepsilon^{1 / 2} V_{L}\left(\alpha^{*}\right) w+O\left(\varepsilon^{1 / 2}|w|^{3}+\varepsilon|w|+\varepsilon^{3 / 2}\right)\right), \\
\dot{\psi}_{i} & =\varepsilon_{n}\left(\left(\alpha_{i}^{*}+w_{i}\right)^{2 m-1}+\varepsilon^{1 / 2} \bar{\Phi}_{i}^{0}\left(\alpha^{*}\right)+O\left(\varepsilon^{1 / 2}|w|+\varepsilon\right)\right) .
\end{aligned}
$$


Дифференцируя, как обычно, (22) в силу систем (21) и (23) и приравнивая слагаемые при $\varepsilon^{1 / 2}$, получаем уравнения $\sum_{\nu=1}^{d} \alpha_{\nu}^{* 2 m-1} \partial \widetilde{\theta}_{i}(\psi) / \partial \psi_{\nu}=\breve{\Phi}_{i}\left(\alpha^{*}, \psi\right)-\bar{\Phi}_{i}^{0}\left(\alpha^{*}\right)$, которые однозначно разрешимы.

Остается сделать масштабирующую замену

$$
w=\varepsilon z
$$

приводящую систему (23) к требуемому виду:

$$
\begin{aligned}
\dot{z} & =\varepsilon_{n}\left(\varepsilon^{1 / 2} V_{L}\left(\alpha^{*}\right) z+\varepsilon^{1 / 2} Z\left(\varepsilon^{1 / 4} z, \psi, \varepsilon\right)\right), \\
\dot{\psi}_{i} & =\varepsilon_{n}\left(\alpha_{i}^{* 2 m-1}+\varepsilon^{1 / 2} \bar{\Phi}_{i}^{0}\left(\alpha^{*}\right)+\varepsilon^{3 / 4} \Psi_{i}\left(\varepsilon^{1 / 4} z, \psi, \varepsilon\right)\right),
\end{aligned}
$$

где $z=\left(z_{1}, \ldots, z_{d}\right) ; Z, \Psi$ - непрерьвные, непрерывно дифференцируемые по $z, \psi_{i}$ при малых $|z|, \varepsilon$ векторные функции, $\omega$-периодические по $\psi_{i}$.

ЗАмЕЧАнИЕ 3 . Функции $Z, \Psi$ из системы (25) будут иметь указанную структуру и гладкость, если потребовать, чтобы в системе $(1)$ при $n=2 m$ функции $\widehat{X}_{i}^{*[n+3]}(x, \varepsilon)$ и $\widehat{X}_{i+d}^{*[2 n+2]}(x, \varepsilon)$ были непрерывными по совокупности аргументов и непрерывно дифференцируемьми по $x_{1}, \ldots, x_{2 d}$ в $H$.

В работе [1, с. 32] для системы (6) с $k=n$ и $\omega=2 \pi$

$$
\dot{z}=\varepsilon^{n} L z+\varepsilon^{n} Z\left(\varepsilon^{1 / 2} z, \psi, \varepsilon\right), \quad \dot{\psi}=\mu(\varepsilon)+\varepsilon^{n+1 / 2} \Psi_{i}\left(\varepsilon^{1 / 2} z, \psi, \varepsilon\right)
$$

при тех же условиях на вектор-функции $Z, \Psi$ и матрицу $L$, что и в системе $(25) ;$ в $[1$, с. 31$]$ доказана теорема, являющаяся одной из модификаций леммы 2.1 работы Хейла [3], согласно которой у системы (6) из [1] при всех достаточно малых положительных значениях $\varepsilon$ существует инвариантное многообразие $z=\Gamma(\psi, \varepsilon)$, где функция $\Gamma$ непрерывная и $\omega$-периодическая по $\psi_{i}$.

Система (25) отличается от системы (6) из [1] только тем, что переменная $z$ в аргументах функций $Z, \Psi$ домножается не на $\varepsilon^{1 / 2}$, а на $\varepsilon^{1 / 4}$, и сама функция $\Psi$ имеет множителем $\varepsilon^{n+1 / 4}$, а не $\varepsilon^{n+1 / 2}$. Однако из доказательства теоремы в [1] легко заметить, что различия в степенях $\varepsilon$ в указанных местах несущественны, и теорема остается справедливой, если $\varepsilon^{1 / 2}$ везде заменить на $\varepsilon^{\gamma}(\gamma>0)$.

Таким образом, система (25) при любом малом значении параметра имеет непрерывное $\omega$-периодическое $d$-мерное инвариантное многообразие $z=\Gamma(\psi, \varepsilon)$.

6. Дополнительное осреднение при $n=2 m-1$. Система $\left(\breve{\boldsymbol{8}}_{\mathrm{od}}\right)$. Вернемся к системе (10), входящие в которую функции $V, \Phi, W$ описаны формулами (14)-(16), и рассмотрим случай, когда $n=2 m-1, m \geqslant 2$. Случай $m=1$ подробно изучен в [ 1 , гл. $1, \S 1]$.

При нечетных $n$ в системе (6)

$$
\begin{aligned}
& R_{i}^{[1]}(\alpha, \varphi, 1)=\operatorname{Cs}^{4 m-3} \varphi_{i} \sum_{\mu=0}^{m} X_{i 2 m}^{[2 \mu]}-\alpha_{i}^{2-2 m} \operatorname{Sn} \varphi_{i} \sum_{\mu=0}^{2 m-1} X_{i+d 4 m-2}^{[2 \mu]}, \\
& R_{i}^{[2]}(\alpha, \varphi, 1)=\operatorname{Cs}^{4 m-3} \varphi_{i} \sum_{\mu=0}^{m} X_{i 2 m+1}^{[2 \mu+1]}-\alpha_{i}^{2-2 m} \operatorname{Sn} \varphi_{i} \sum_{\mu=0}^{2 m-1} X_{i+d 4 m-1}^{[2 \mu+1]}, \\
& \Phi_{i}^{[1]}(\alpha, \varphi, 1)=(1-2 m) \operatorname{Sn} \varphi_{i} \sum_{\mu=0}^{m} X_{i 2 m}^{[2 \mu]}-\alpha_{i}^{2-2 m} \operatorname{Cs} \varphi_{i} \sum_{\mu=0}^{2 m-1} X_{i+d 4 m-2}^{[2 \mu]},
\end{aligned}
$$


где $X_{\iota k}^{[\varkappa]}$ имеют аргументы $\alpha \operatorname{Cs} \varphi,-\alpha^{2 m-1} \operatorname{Sn} \varphi$ и порядок $\varkappa$ по $\alpha_{1}, \ldots, \alpha_{d}$.

Нетрудно убедится в том, что любое слагаемое в $R_{i}^{[1]}(\alpha, \varphi, 1)$ из $(26)$ содержит какой-либо из $\operatorname{Sn} \varphi_{j}$ или $\operatorname{Cs} \varphi_{j}$ в нечетной степени, а значит, имеет нулевое среднее значение. Следовательно, при любых $\alpha$ из замены (7), удовлетворяюших (13), в формуле (14) $V_{i}(\alpha) \equiv 0$, что приводит к вырождению бифуркационной системы и аннулированию в системе (10) не только свободных, но и линейных по $v$ членов, стоящих при $\varepsilon_{n} \varepsilon^{1 / 2}$.

В результате система (10) при нечетных $n$ принимает вид

$$
\begin{aligned}
& \dot{v}_{i}=\varepsilon_{n}\left(\varepsilon W_{i}(\alpha, \varphi)+\varepsilon \sum_{j=1}^{d} W_{i}^{(j)}(\alpha, \varphi) v_{j}+O\left(\varepsilon^{1 / 2}|v|^{3}+\varepsilon|v|^{2}+\varepsilon^{3 / 2}\right)\right), \\
& \dot{\varphi}_{i}=\varepsilon_{n}\left(\left(\alpha_{i}+v_{i}\right)^{2 m-2}+O\left(\varepsilon^{1 / 2}\right)\right) .
\end{aligned}
$$

Для того чтобы свести ситуацию к стандартной при $n=2 m-1$, придется сделать дополнительную замену, осредняющую теперь уже функцию $W(\alpha, \varphi)$ из $(16)$, в которой согласно (6) задействованы не только однородные полиномы $X_{i}^{[n+1]}(x, \varepsilon)$ и $X_{i+d}^{[2 n]}(x, \varepsilon)$, но и полиномы старших порядков $X_{i}^{[n+2]}(x, \varepsilon)$ и $X_{i+d}^{[2 n+1]}(x, \varepsilon)$ системы $(1)$.

Покажем, что существует осредняющая замена переменных

$$
v_{i}=\breve{v}_{i}+\varepsilon \widetilde{\chi}_{i}(\alpha, \varphi)+\varepsilon \sum_{j=1}^{d} \widetilde{\chi}_{i}^{(j)}(\alpha, \varphi) \breve{v}_{j}, \quad i=1, \ldots, d,
$$

переводящая систему $\left(10_{\text {od }}\right)$ в систему

$$
\begin{aligned}
& \dot{\vec{v}}_{i}=\varepsilon_{n}\left(\varepsilon \breve{V}_{i}(\alpha)+\varepsilon \sum_{j=1}^{d} \breve{V}_{i}^{(j)}(\alpha) \breve{v}_{j}+O\left(\varepsilon^{1 / 2}|\breve{v}|^{3}+\varepsilon|\breve{v}|^{2}+\varepsilon^{3 / 2}\right)\right), \\
& \dot{\varphi}_{i}=\varepsilon_{n}\left(\left(\alpha_{i}+\breve{v}_{i}\right)^{2 m-2}+O\left(\varepsilon^{1 / 2}\right)\right) .
\end{aligned}
$$

Продифференцируем замену $(9) \quad v=\breve{v}+\varepsilon \widetilde{\chi}(\breve{v}, \alpha, \varphi)$ в силу систем $\left(10_{\text {od }}\right)$ и $\left(\breve{8}_{\text {od }}\right)$ и сократим полученные равенства на $\varepsilon_{n}$; тогда

$$
\begin{aligned}
\varepsilon W_{i}+\varepsilon & \sum_{j=1}^{d} W_{i}^{(j)}\left(\breve{v}_{j}+\varepsilon^{1 / 2} \widetilde{\chi}_{j}\right)+O\left(\varepsilon^{1 / 2}|\breve{v}|^{3}+\varepsilon|\breve{v}|^{2}+\varepsilon^{3 / 2}\right) \\
= & \varepsilon \breve{V}_{i}+\varepsilon \sum_{j=1}^{d} \breve{V}_{i}^{(j)} \breve{v}_{j}+\varepsilon \sum_{j=1}^{d} \widetilde{\chi}_{i}^{(j)} \cdot O\left(\varepsilon^{1 / 2}\right) \\
& +\varepsilon \sum_{\nu=1}^{d}\left(\frac{\partial \widetilde{\chi}_{i}}{\partial \varphi_{\nu}}+\sum_{j=1}^{d} \breve{v}_{j} \frac{\partial \widetilde{\chi}_{i}^{(j)}}{\partial \varphi_{\nu}}\right)\left(\left(\alpha_{\nu}+\breve{v}_{\nu}\right)^{2 m-2}+O\left(\varepsilon^{1 / 2}\right)\right) .
\end{aligned}
$$

Приравнивая слагаемые, стоящие при $\varepsilon$, получаем уравнения

$$
\sum_{\nu=1}^{d} \frac{\partial \widetilde{\chi}_{i}(\alpha, \varphi)}{\partial \varphi_{\nu}} \alpha_{\nu}^{2 m-2}=W_{i}(\alpha, \varphi)-\breve{V}_{i}(\alpha), \quad i=1, \ldots, d .
$$

Аналогично (12) уравнения (28) однозначно разрешимы относительно $\widetilde{\chi}_{i}$, имеющих нулевые средние значения, при уже вьполненом предположении (13) на вектор $\alpha$ и при

$$
\breve{V}_{i}(\alpha)=\overline{W_{i}(\alpha, \varphi)}=\omega^{-d} \int_{0}^{\omega} \ldots \int_{0}^{\omega} W_{i}(\alpha, \varphi) d \varphi_{1} \ldots d \varphi_{d} .
$$

А приравнивая слагаемые, стоящие при $\varepsilon \breve{v}_{j}$, получаем такие же уравнения, как при дифференцировании (28) по $\alpha_{j}$. Следовательно, дополнительная осредняющая замена (27) построена. 
7. Бифуркационная система для нечетных $n$. Система $\left(\breve{8}_{\text {od }}^{*}\right)$. Подсчитаем $\breve{V}_{i}(\alpha)$. Согласно $(16),(15)$ и с учетом того, что $V_{i}(\alpha) \equiv 0$ при $n=2 m-1(m \geqslant 2)$, имеем

$$
\begin{gathered}
W_{i}(\alpha, \varphi)=R_{i}^{[2]}(\alpha, \varphi, 1)+P_{i}^{[1]}(\alpha, \varphi) \\
P_{i}^{[1]}=\sum_{j=1}^{d} R_{i}^{[1](j)}(\alpha, \varphi, 1) \tilde{f}_{j}-\sum_{\nu=1}^{d} \frac{\partial \tilde{f}_{i}}{\partial \varphi_{\nu}}\left((2 m-2) \alpha_{\nu}^{2 m-3} \tilde{f}_{\nu}+\alpha_{\nu}^{-1} \Phi_{\nu}^{[1]}(\alpha, \varphi, 1)\right)
\end{gathered}
$$

функции $\tilde{f}_{i}$ определены в $(12)$, а $R_{i}^{[1]}, R_{i}^{[2]}, \Phi_{i}^{[1]}$ в (26).

Следовательно, функции $P_{i}^{[1]}(\alpha, \varphi)$ зависят только от однородных полиномов $X_{i}^{[n+1]}(x, \varepsilon), X_{i+d}^{[2 n]}(x, \varepsilon), \quad n=2 m-1$, системы (1) и тождественно равны нулю, если эти полиномы отсутствуют. Из (29) видно, что $P_{i}^{[1]}$ могут содержать как положительные, так и отрицательные степени $\alpha$.

В свою очередь, для вычисления среднего значения из $R_{i}^{[2]}(\alpha, \varphi, 1)$ выделим те слагаемые, которые содержат только четные степени обобщенных синусов и косинусов, обозначив их $R_{i, \mathrm{ev}}^{[2]}(\alpha, \varphi)$. При $n=2 m-1$ имеем

$$
R_{i, \mathrm{ev}}^{[2]}=\alpha_{i} \sum_{\mu=0}^{m} \sum_{l:|l|=\mu}\left(\mathrm{Cs}^{4 m-2} \varphi_{i} X_{i 2 m+1}^{\left(2 l+e_{i}, 0\right)}+\operatorname{Sn}^{2} \varphi_{i} X_{i+d 4 m-1}^{\left(2 l, e_{i}\right)}\right) \prod_{j=1}^{d} \alpha_{j}^{2 l_{j}} \mathrm{Cs}^{2 l_{j}} \varphi_{j} .
$$

Учитывая, что $\overline{R_{i}^{[2]}}=\overline{R_{i, \mathrm{ev}}^{[2]}}$, аналогично $\left(14_{\mathrm{ev}}\right)$ найдем $\breve{V}_{i}$ из $(\breve{12})$ :

$$
\breve{V}_{i}(\alpha)=\alpha_{i} \sum_{l:|l|=0}^{m} \frac{\varkappa_{n}^{(l)}}{n+2 l_{i}+1}\left(\left(2 l_{i}+1\right) X_{i 2 m+1}^{\left(2 l+e_{i}, 0\right)}+X_{i+d 4 m-1}^{\left(2 l, e_{i}\right)}\right) \alpha^{2 l}+\overline{P_{i}^{[1]}(\alpha, \varphi)},\left(\breve{12_{\mathrm{od}}}\right)
$$

где функция $P_{i}^{[1]}$ определена в $(29), n=2 m-1(m \geqslant 2)$.

ОПРЕДЕЛЕНИЕ 2. Алгебраическая система $\breve{V}(\alpha)=0$, где векторньй полином $\breve{V}$ задан формулой $(\breve{12}$ оd $)$, называется бифуркационной системой уравнений для системы (1), в которой $n=2 m-1$.

ЗАмЕчаниЕ 4 . При $n=2 m-1, m \geqslant 2$, если однородные полиномы $X_{i}^{[n+1]}(x, \varepsilon)$, $X_{i+d}^{[2 n]}(x, \varepsilon) \equiv 0$, т.е. в $(29) P_{i}^{[1]}(\alpha, \varphi) \equiv 0$ и $W_{i}=R_{i}^{[2]}$, то бифуркационная система - это алгебраическая система $d$ уравнений степени $m$ относительно $\alpha_{1}^{2}, \ldots, \alpha_{d}^{2}$, определяемая коэффициентами полиномов $X_{i}^{[n+2]}(x, \varepsilon)$ и $X_{i+d}^{[2 n+1]}(x, \varepsilon)$. Если же $P_{i}^{[1]}(\alpha, \varphi) \neq 0$, то бифуркационная система может иметь более высокую степень и, соответственно, большее число положительных решений.

По аналогии с $V_{L}$ введем матрищу $\breve{V}_{L}(\alpha)=\left\{\breve{V}_{i}^{(j)}(\alpha)\right\}_{i, j=1}^{d}$ и для $\breve{V}, \breve{V}_{L}$ сфформулируем условия, аналогичные условиям (18). Предположим, что $\exists \alpha^{*}=\left(\alpha_{1}^{*}, \ldots, \alpha_{d}^{*}\right)>0$ :

1) $\breve{V}\left(\alpha^{*}\right)=0$

2) $\alpha^{*}$ удовлетворяет условию (13);

3) $\breve{V}_{L}\left(\alpha^{*}\right)$ - некритическая матрища.

Для проверки условия $\left(1_{3}\right)$ матрицу $\breve{V}_{L}\left(\alpha^{*}\right)$ легко подсчитать, дифференцируя равенство $(\breve{12}$ од $)$ по $\alpha_{j}$ в точке $\alpha^{*}$.

В результате система $\left(\breve{8}_{\text {od }}\right)$, в которой $n=2 m-1$ и $\alpha=\alpha^{*}$ из $(18)$, принимает вид

$$
\begin{aligned}
\dot{\vec{v}} & =\varepsilon_{n}\left(\varepsilon \breve{V}_{L}\left(\alpha^{*}\right) \breve{v}+O\left(\varepsilon^{1 / 2}|\breve{v}|^{3}+\varepsilon|\breve{v}|^{2}+\varepsilon^{3 / 2}\right)\right), \\
\dot{\varphi}_{i} & =\varepsilon_{n}\left(\left(\alpha_{i}^{*}+\breve{v}_{i}\right)^{2 m-2}+O\left(\varepsilon^{1 / 2}\right)\right) .
\end{aligned}
$$


8. Процесс осреднения системы $\left(\breve{8}_{\mathrm{od}}^{*}\right)$. Система $(35)$. К системе $\left(\breve{8}_{\mathrm{od}}^{*}\right)$ непосредственно нельзя применить лемму Хейла. Чтобы достичь требуемой структуры, в $\left(\breve{8}_{\text {od }}^{*}\right)$ надо осреднить члены, зависяшие только от $\varphi$, при младших степенях $\varepsilon$, используя замены, аналогичные заменам $(20),(22),(24)$. Но из-за того, что в $\left(\breve{8}_{\text {od }}^{*}\right)$ в уравнениях для $\dot{\vec{v}}$ линейные по $\breve{v}$ члены имеют больший порядок $\varepsilon$, чем такие же члены в $\left(10_{\text {ev }}^{*}\right)$, потребуется осреднять большее число слагаемых.

Покажем сначала, что существует замена

$$
\breve{v}=w-\varepsilon^{1 / 2} \bar{g}\left(\alpha^{*}\right)-\varepsilon \bar{h}\left(\alpha^{*}\right)+\varepsilon^{3 / 2} \tilde{g}\left(\alpha^{*}, \varphi\right)+\varepsilon^{2} \widetilde{h}\left(\alpha^{*}, \varphi\right),
$$

которая преобразует $\left(\breve{8}_{\text {od }}^{*}\right)$ в систему

$$
\begin{aligned}
\dot{w} & =\varepsilon_{n}\left(\varepsilon \breve{V}_{L}\left(\alpha^{*}\right) w+O\left(\varepsilon^{1 / 2}|w|^{3}+\varepsilon|w|^{2}+\varepsilon^{3 / 2}|w|+\varepsilon^{5 / 2}\right)\right), \\
\dot{\varphi}_{i} & =\varepsilon_{n}\left(\left(\alpha_{i}^{*}+w_{i}\right)^{2 m-2}+\varepsilon^{1 / 2} b_{i}^{(1)}(\varphi)+\varepsilon b_{i}^{(2)}(\varphi)+O\left(\varepsilon^{1 / 2}|w|+\varepsilon^{3 / 2}\right)\right),
\end{aligned}
$$

где $b_{i}^{(1)}(\varphi), b_{i}^{(2)}(\varphi)$ - это функции при соответствующих степенях $\varepsilon$, выделенные в уравнениях для $\dot{\varphi}_{i}$ системы $\left(\breve{8}_{\mathrm{od}}^{*}\right)$ из функций $O\left(\varepsilon^{1 / 2}\right)$ после подстановки в них замены $(30)$.

В свою очередь, функцию $O\left(\varepsilon^{1 / 2}|\breve{v}|^{3}+\varepsilon|\breve{v}|^{2}+\varepsilon^{3 / 2}\right)$ системы $\left(\breve{8}_{\text {оd }}^{*}\right)$ после подстановки в нее замены (30) можно записать следующим образом:

$$
O\left(\varepsilon^{1 / 2}|\breve{v}|^{3}+\cdots\right)=\varepsilon^{3 / 2} b^{(3)}(\varphi)+\varepsilon^{2} b^{(4)}(\varphi)+O\left(\varepsilon^{1 / 2}|w|^{3}+\varepsilon|w|^{2}+\varepsilon^{3 / 2}|w|+\varepsilon^{5 / 2}\right) .
$$

Поэтому после дифференцирования замены (30) в силу систем $\left(\breve{8}_{\text {od }}^{*}\right)$ и $(31)$, а также сокращения на $\varepsilon_{n}$, получаем равенство

$$
\begin{aligned}
\varepsilon \breve{V}_{L}\left(\alpha^{*}\right)(w- & \left.\varepsilon^{1 / 2} \bar{g}-\varepsilon \bar{h}+\varepsilon^{3 / 2} \tilde{g}+\varepsilon^{2} \widetilde{h}\right)+\varepsilon^{3 / 2} b^{(3)}+\varepsilon^{2} b^{(4)} \\
= & \varepsilon \breve{V}_{L}\left(\alpha^{*}\right) w+O\left(\varepsilon^{1 / 2}|w|^{3}+\varepsilon|w|^{2}+\varepsilon^{3 / 2}|w|+\varepsilon^{5 / 2}\right) \\
& +\sum_{\nu=1}^{d}\left(\varepsilon^{3 / 2} \frac{\partial \tilde{g}}{\partial \varphi_{\nu}}+\varepsilon^{2} \frac{\partial \widetilde{h}}{\partial \varphi_{\nu}}\right) \\
& \times\left(\left(\alpha_{\nu}^{*}+w_{\nu}\right)^{2 m-2}+\varepsilon^{1 / 2} b_{\nu}^{(1)}+\varepsilon b_{\nu}^{(2)}+O\left(\varepsilon^{1 / 2}|w|+\varepsilon^{3 / 2}\right)\right) .
\end{aligned}
$$

Приравняем слагаемые при $\varepsilon^{3 / 2}$ и $\varepsilon ;$ тогда

$$
\sum_{\nu=1}^{d} \frac{\partial \tilde{g}}{\partial \varphi_{\nu}} \alpha_{\nu}^{* 2 m-2}=b^{(3)}-\breve{V}_{L} \bar{g}, \quad \sum_{\nu=1}^{d} \frac{\partial \widetilde{h}}{\partial \varphi_{\nu}} \alpha_{\nu}^{* 2 m-2}=b^{(4)}-\sum_{\nu=1}^{d} \frac{\partial \tilde{g}}{\partial \varphi_{\nu}} b_{\nu}^{(1)}-\breve{V}_{L} \bar{h} .
$$

Полученные уравнения стандартньм образом однозначно разрешимы благодаря условиям $\left(18_{2}\right),\left(18_{3}\right)$, и замена (30) построена.

ЗАмЕчАНИЕ 5. Именно для того чтобы было возможно осуществить замену (30), в системе (8) пришлось осреднить члены при $\varepsilon_{n} \varepsilon^{1 / 2} v^{2}$, получая $O\left(\varepsilon^{1 / 2}|v|^{3}+\cdots\right)$ в $\left(10_{\text {od }}\right)$, иначе величины $\breve{V}_{i}\left(\alpha^{*}\right)$ были бы испорчены.

Осредним теперь функции $b^{(1)}, b^{(2)}$, входящие в $(31)$, сделав замену

$$
\dot{\varphi}_{i}=\psi_{i}+\varepsilon^{1 / 2} \tilde{\theta}_{i}(\psi)+\varepsilon \tilde{\eta}_{i}(\psi),
$$


которая преобразует систему (31) в систему

$$
\begin{aligned}
\dot{w} & =\varepsilon_{n}\left(\varepsilon \breve{V}_{L}\left(\alpha^{*}\right) w+O\left(\varepsilon^{1 / 2}|w|^{3}+\varepsilon|w|^{2}+\varepsilon^{3 / 2}|w|+\varepsilon^{5 / 2}\right)\right) \\
\dot{\psi}_{i} & =\varepsilon_{n}\left(\left(\alpha_{i}^{*}+w_{i}\right)^{2 m-2}+\varepsilon^{1 / 2} \bar{b}_{i}^{(1)}+\varepsilon \breve{b}_{i}^{(2)}+O\left(\varepsilon^{1 / 2}|w|+\varepsilon^{3 / 2}\right)\right) .
\end{aligned}
$$

Дифференцируя (32) в силу (31) и (33) и сокращая на $\varepsilon_{n}$, получаем

$$
\begin{aligned}
& \varepsilon^{1 / 2} b_{i}^{(1)}\left(\psi+\varepsilon^{1 / 2} \tilde{\theta}+\varepsilon \tilde{\eta}\right)+\varepsilon b_{i}^{(2)}(\psi)+O\left(\varepsilon^{1 / 2}|w|+\varepsilon^{3 / 2}\right)=\varepsilon^{1 / 2} \bar{b}_{i}^{(1)}+\varepsilon \breve{b}_{i}^{(2)} \\
& +\sum_{\nu=1}^{d}\left(\varepsilon^{1 / 2} \frac{\partial \tilde{\theta}_{i}}{\partial \psi_{\nu}}+\varepsilon \frac{\partial \tilde{\eta}_{i}}{\partial \psi_{\nu}}\right)\left(\left(\alpha_{\nu}^{*}+w_{\nu}\right)^{2 m-2}+\varepsilon^{1 / 2} \bar{b}_{\nu}^{(1)}+O\left(\varepsilon^{1 / 2}|w|+\varepsilon\right)\right)
\end{aligned}
$$

откуда

$$
\begin{aligned}
& \sum_{\nu=1}^{d} \frac{\partial \tilde{\theta}_{i}(\psi)}{\partial \psi_{\nu}} \alpha_{\nu}^{* 2 m-2}=b_{i}^{(1)}(\psi)-\bar{b}_{i}^{(1)} \\
& \sum_{\nu=1}^{d} \frac{\partial \tilde{\eta}_{i}(\psi)}{\partial \psi_{\nu}} \alpha_{\nu}^{* 2 m-2}=b_{i}^{(2)}(\psi)+\sum_{\nu=1}^{d}\left(\frac{\partial b_{i}^{(1)}(\psi)}{\partial \psi_{\nu}} \tilde{\theta}_{\nu}(\psi)-\frac{\partial \tilde{\theta}_{i}(\psi)}{\partial \psi_{\nu}} \bar{b}_{\nu}^{(1)}\right)-\breve{b}_{i}^{(2)}
\end{aligned}
$$

и величины $\bar{b}^{(1)}\left(\alpha^{*}\right), \breve{b}^{(2)}\left(\alpha^{*}\right), \tilde{\theta}_{i}(\psi), \tilde{\eta}_{i}(\psi)$ могут быть однозначно найдены.

Остается сделать масштабирующую замену

$$
w=\varepsilon^{3 / 2} z
$$

приводящую систему (33) к виду

$$
\begin{aligned}
\dot{z} & =\varepsilon_{n}\left(\varepsilon^{1 / 2} \breve{V}_{L}\left(\alpha^{*}\right) z+\varepsilon Z\left(\varepsilon^{1 / 4} z, \psi, \varepsilon\right)\right) \\
\dot{\psi}_{i} & =\varepsilon_{n}\left(\alpha_{i}^{* 2 m-2}+\varepsilon^{1 / 2} \bar{b}_{i}^{(1)}\left(\alpha^{*}\right)+\varepsilon \breve{b}_{i}^{(2)}\left(\alpha^{*}\right)+\varepsilon^{5 / 4} \Psi_{i}\left(\varepsilon^{1 / 4} z, \psi, \varepsilon\right)\right)
\end{aligned}
$$

где $z=\left(z_{1}, \ldots, z_{d}\right) ; Z, \Psi$ - непрерьвные, непрерывно дифференцируемые по $z, \psi_{i}$ при малых $|z|, \varepsilon$ векторные функции, $\omega$-периодические по $\psi_{i}$.

ЗАмЕЧАниЕ 6 . Функции $Z, \Psi$ из системы (25) будут иметь указанную структуру и гладкость, если потребовать, чтобы в (1) при $n=2 m-1$ функции $X_{i}^{*[n+3]}(x, \varepsilon)$ и $X_{i+d}^{*[2 n+2]}(x, \varepsilon)$ были представимы в виде

$$
X_{i}^{*[n+3]}=X_{i}^{[n+3]}+X_{i}^{[n+4]}+X_{i}^{*[n+5]}, \quad X_{i+d}^{*[2 n+2]}=X_{i+d}^{[2 n+2]}+X_{i+d}^{[2 n+3]}+X_{i+d}^{*[2 n+4]},
$$

где $X_{\iota}^{*[k]}\left(\varepsilon^{1 / 2} x^{\prime}, \varepsilon^{n / 2} x^{\prime \prime}, \varepsilon\right)=\varepsilon^{k} \widehat{X}_{\iota}^{*[k]}\left(x^{\prime}, x^{\prime \prime}, \varepsilon\right), \iota=1, \ldots, 2 d$, а $\widehat{X}_{i}^{*[n+5]}$ и $\widehat{X}_{i+d}^{*[2 n+4]}$ непрерьвны и непрерывно дифференцируемы по $x_{1}, \ldots, x_{2 d}$.

Система (35), как и (25), имеет требуемую в лемме Хейла структуру, поэтому у нее при всех достаточно малых $\varepsilon>0$ существует $d$-мерное инвариантное многообразие $z=$ $\Gamma(\psi, \varepsilon)$, где функция $\Gamma$ - непрерывная и $\omega$-периодическая по $\psi_{i}$. 
9. Существование и структура инвариантных торов. Возвращаясь к системе (1), в которой гладкость отмеченных звездочкой функций уточнена в замечаниях 3 и 6 , заключаем, что как при четных $n$, когда вьполняется условие (18) для $\alpha=\alpha^{*}$ из замены $(7), V\left(\alpha^{*}\right)$ из $\left(14_{\mathrm{ev}}\right)$ и $V_{L}\left(\alpha^{*}\right)$ из $(19)$, так и при нечетных $n$, когда выполняется условие (16) с $\breve{V}\left(\alpha^{*}\right)$ из $(\breve{12}$ оd $)$, система (1) обладает при всех малых положительных значениях параметра инвариантньми поверхностями, которые заменой (5) свернуты в $d$-мерные торы, т.е. справедливо следующее утверждение.

Теорема 1. Предположим, что для системы (1) в случае $n=2 m, m \geqslant 1$, выполняется условие (18), а в случае $n=2 m-1, m \geqslant 2$, выполняется условие (16). Тогда при любом достаточно малом $\varepsilon>0$ эта система имеет $d$-мерный инвариантный тор с частотами движений на нем близкими $\kappa \varepsilon^{n / 2-1 / 2} \alpha_{i}^{* n-1} \omega^{-1}$, $i=1, \ldots, d$.

ЗАмЕчАнИЕ 7. В условиях (18), (年), гарантирующих при каждом малом $\varepsilon>0$ сушествование хотя бы одного инвариантного тора, используется конечное число коэффициентов исходной системы (1) и вьполнение этих условий может быть непосредственно проверено. При этом определенная в $\left(14_{\mathrm{ev}}\right)$ функция $V(\alpha)$, которая при четных $n$ задает бифуркационную систему $V(\alpha)=0$, зависит только от полиномов $X_{i}^{[n+1]}, X_{i+d}^{[2 n]}$ системы $(1)$, а функция $\breve{V}(\alpha)$, при нечетных $n$ определенная в $\left(\breve{12}{ }_{\text {od }}\right)$, зависит как от указанньх полиномов, так и от полиномов $X_{i}^{[n+2]}, X_{i+d}^{[2 n+1]}$.

ЗАмЕчАниЕ 8. Если правые части (1) не содержат функций, зависящих только от $\varepsilon$, то можно говорить о бифуркации рождения $d$-мерного инвариантного тора, ибо нулевое решение сохраняется при всех $\varepsilon>0$.

ЗАмЕЧАниЕ 9. Система (1) при каждом $\varepsilon$ может обладать несколькими $d$-мерньми инвариантными торами, количество которых зависит от числа положительных решений $\alpha^{* 2}$ бифуркационной системы, удовлетворяющих, например, при четных $n$ условиям $\left(18_{2}\right)$ и $\left(18_{3}\right)$. Если жепри каком-то $\alpha^{*}$ условие $\left(18_{3}\right)$ нарушается, можно продолжать процесс осреднения теперь уже системы (25) в поисках у системы (1) инвариантных торов большей размерности, как это делалось в сходных ситуациях в работах [5, гл. 1] и [2]. Те же рассуждения верны и для нечетных $n$.

Поскольку доказательство теоремы 1 конструктивно, во всяком случае при четных $n$ непосредственно для системы (1) можно в явном виде выписать главные по $\varepsilon$ члены инвариантной поверхности, тем самым, указав ее структуру. При нечетных $n$ формулы становятся менее наглядными.

СлЕДСТВИЕ 1. Пусть в системе (1) $n=2 m$ и выполняется условие (18). Тогда для любого достаточно малого $\varepsilon>0$ инвариантная поверхность системь (1), наличие которой гарантирует теорема 1 , имеет вид

$$
\begin{gathered}
x^{\prime}=\varepsilon^{1 / 2} \alpha^{*} \operatorname{Cs} \psi+\varepsilon\left((\tilde{f}(\psi)-\bar{g}) \operatorname{Cs} \psi-\alpha^{*} \tilde{\theta}(\psi) \operatorname{Sn} \psi\right)+O\left(\varepsilon^{3 / 2}\right), \\
x^{\prime \prime}=-\varepsilon^{m} \alpha^{* 2 m} \operatorname{Sn} \psi-\varepsilon^{m+1 / 2}\left(2 m \alpha^{* 2 m-1}(\tilde{f}(\psi)-\bar{g}) \operatorname{Sn} \psi\right. \\
\left.\quad+\alpha^{* 2 m} \tilde{\theta}(\psi) \operatorname{Cs}^{4 m-1} \psi\right)+O\left(\varepsilon^{m+1}\right),
\end{gathered}
$$

где вектор $\alpha^{*}$ из замены (7) удовлетворяет условию (18), $\omega$ - периодические функиии $\tilde{f}(\psi)$ и $\tilde{\theta}(\psi)$ определены в заменах $\left(9_{\mathrm{ev}}\right) u(22)$, а $\bar{g}-$ в замене $(20), \operatorname{Cs} \varphi и \operatorname{Sn} \varphi$ 
используются в замене (5). При этом функиия $\Gamma(\psi, \varepsilon)$, задающая инвариантная поверхность, существование которой для системы (25) гарантируется леммой Хейла, входит в первое уравнение (36), имея мнохитель $\varepsilon^{3 / 2}$, а во второе мнохситель $\varepsilon^{m+1}$.

В самом деле, при $n=2 m, m \geqslant 2$, суперпозиция шести замен $(5),(7),\left(9_{\mathrm{ev}}\right),(20)$, $(22),(24)$, где в последней вместо переменной $z$ подставлена функция $\Gamma(\psi, \varepsilon)$ из леммы Хейла, представляет собой инвариантньй тор системы (1), имеющий вид

$$
\begin{aligned}
x^{\prime}= & \varepsilon^{1 / 2}\left(\alpha^{*}+\varepsilon \Gamma-\varepsilon^{1 / 2} \bar{g}+\varepsilon \tilde{g}\left(\psi+\varepsilon^{1 / 2} \tilde{\theta}\right)+\varepsilon^{1 / 2} \tilde{f}\left(\psi+\varepsilon^{1 / 2} \tilde{\theta}\right)\right. \\
& \left.+\varepsilon^{1 / 2} \tilde{f}_{L}\left(\psi+\varepsilon^{1 / 2} \tilde{\theta}\right) \cdot\left(\varepsilon \Gamma-\varepsilon^{1 / 2} \bar{g}+\varepsilon \tilde{g}\left(\psi+\varepsilon^{1 / 2} \tilde{\theta}\right)\right)\right) \operatorname{Cs}\left(\psi+\varepsilon^{1 / 2} \tilde{\theta}\right), \\
x^{\prime \prime}=- & \varepsilon^{n / 2}\left(\alpha^{*}+\varepsilon \Gamma-\varepsilon^{1 / 2} \bar{g}+\varepsilon \tilde{g}\left(\psi+\varepsilon^{1 / 2} \tilde{\theta}\right)+\varepsilon^{1 / 2} \tilde{f}\left(\psi+\varepsilon^{1 / 2} \tilde{\theta}\right)\right. \\
& \left.+\varepsilon^{1 / 2} \tilde{f}_{L}\left(\psi+\varepsilon^{1 / 2} \tilde{\theta}\right) \cdot\left(\varepsilon \Gamma-\varepsilon^{1 / 2} \bar{g}+\varepsilon \tilde{g}\left(\psi+\varepsilon^{1 / 2} \tilde{\theta}\right)\right)\right)^{n} \operatorname{Sn}\left(\psi+\varepsilon^{1 / 2} \tilde{\theta}\right) .
\end{aligned}
$$

Выделяя в правых частях полученных равенств члены, стояшие при двух младших степенях параметра $\varepsilon$, получаем уравнения (36), в которых, как обычно, произведение, например, векторов $\alpha^{* 2 m} \tilde{\theta}(\psi) \mathrm{Cs}^{4 m-1} \psi$ означает вектор с компонентами $\alpha_{i}^{* 2 m} \tilde{\theta}_{i}(\psi) \mathrm{Cs}^{4 m-1} \psi_{i}$.

10. Первый частный случай. Система (37). Применим полученные выше результаты для случая, когда в системе (1) $n=2$, т.е. $n=2 m$ при $m=1$. Тогда переменные $x^{\prime \prime}$ и параметр $\varepsilon$ имеют второй порядок малости, а переменные $x^{\prime}$ - первый.

Система (1) с учетом замечания 3 принимает вид

$$
\begin{aligned}
\dot{x}_{i} & =x_{i+d}+X_{i}^{[3]}(x, \varepsilon)+X_{i}^{[4]}(x, \varepsilon)+X_{i}^{*[5]}(x, \varepsilon), \\
\dot{x}_{i+d} & =-x_{i}^{3}+X_{i+d}^{[4]}(x, \varepsilon)+X_{i+d}^{[5]}(x, \varepsilon)+X_{i+d}^{*[6]}(x, \varepsilon),
\end{aligned}
$$

где $x=\left(x_{1}, \ldots, x_{2 d}\right), X_{\iota}^{[k]}(x, \varepsilon)$ - однородные полиномы порядка $k$ по $x$ и $\varepsilon$ в указанном выше смысле, $\iota=1, \ldots, 2 d$; функции $X_{\iota}^{*[k]}\left(\varepsilon^{1 / 2} x^{\prime}, \varepsilon x^{\prime \prime}, \varepsilon\right)=\varepsilon^{k} \widehat{X}_{\iota}^{*[k]}(x, \varepsilon)$, причем $\widehat{X}_{\iota}^{*[k]}$ - непрерьвные и непрерьвно дифференцируемые по $x_{1}, \ldots, x_{2 d}$ функции.

Таким образом, согласно обозначениям (2)

$$
\begin{aligned}
& X_{i}^{[3]}=X_{i 3}^{[1]}(x) \varepsilon+X_{i 3}^{[3]}(x), \quad X_{i+d}^{[4]}=X_{i+d 4}^{[0]} \varepsilon^{2}+X_{i+d 4}^{[2]}(x) \varepsilon+X_{i+d 4}^{[4]}(x), \\
& X_{\iota 3}^{[1]}=\sum_{j=1}^{d} X_{\iota 3}^{\left(e_{j}, 0\right)} x_{j}, \quad X_{\iota 3}^{[3]}=\sum_{|l|=3} X_{\iota 3}^{(l, 0)} x^{\prime l}+\sum_{j, k=1}^{d} X_{\iota 3}^{\left(e_{j}, e_{k}\right)} x_{j} x_{k+d}, \\
& X_{\iota 4}^{[0]}=c_{0}, \quad X_{\iota 4}^{[2]}=\sum_{|l|=2} X_{\iota 4}^{(l, 0)} x^{\prime l}+\sum_{k=1}^{d} X_{\iota 4}^{\left(0, e_{k}\right)} x_{k+d}, \\
& X_{\iota 4}^{[4]}=\sum_{|l|=4} X_{\iota 4}^{(l, 0)} x^{\prime l}+\sum_{|l|=2} \sum_{k=1}^{d} X_{\iota 4}^{\left(l, e_{k}\right)} x^{\prime l}+\sum_{|l|=2} X_{\iota 4}^{(0, l)} x^{\prime \prime l} .
\end{aligned}
$$

Но далеко не все из вьписанных коэффициентов задействованы в предположениях (18) теоремы 1 и оказьвают влияние на существование инвариантных торов у системы (37). 
В полярной замене $(5)$, когда $n=2, \operatorname{Cs} \varphi_{i}$ и $\operatorname{Sn} \varphi_{i}$ представляют собой эллиптические функции при модуле $2^{-1 / 2}: \operatorname{Cs} \varphi_{i}=\operatorname{cn} \varphi_{i}, \operatorname{Sn} \varphi_{i}=\operatorname{dn} \varphi_{i}$. Их период $\omega=4 \sqrt{2} \int_{0}^{1}(1-$ $\left.\zeta^{4}\right)^{-1 / 2} d \zeta$ и $\operatorname{Cs} \varphi_{i}^{4}+2 \operatorname{Sn} \varphi_{i}^{2}=1$.

Кроме того, при $m=1$ в $\left(14_{\mathrm{ev}}\right) \varkappa_{2}^{0}=1, \varkappa_{2}^{\left(e_{i}\right)}=\omega^{-1} \int_{0}^{\omega} \mathrm{Cs}^{2} \varphi_{i} d \varphi_{i}=v$.

Введем матрицу $A=\left\{a_{i j}\right\}_{i, j=1}^{d}$ и вектор $b=\left(b_{1}, \ldots, b_{d}\right)$, у которых

$$
\begin{array}{ll}
a_{i j}=(v / 3)\left(X_{i 3}^{\left(e_{i}+2 e_{j}, 0\right)}+X_{i+d 4}^{\left(2 e_{j}, e_{i}\right)}\right), & \text { если } i \neq j, \\
a_{i i}=(v / 5)\left(3 X_{i 3}^{\left(3 e_{i}, 0\right)}+X_{i+d 4}^{\left(2 e_{i}, e_{i}\right)}\right), & b_{i}=(1 / 3)\left(X_{i 3}^{\left(e_{i}, 0\right)}+X_{i+d 4}^{\left(0, e_{i}\right)}\right) .
\end{array}
$$

Пусть также матрица $E_{\alpha}=\operatorname{diag}\left\{\alpha_{1}, \ldots, \alpha_{d}\right\}$, вектор $\alpha^{2}=\left(\alpha_{1}^{2}, \ldots, \alpha_{d}^{2}\right)$. Тогда формула $\left(14_{\mathrm{ev}}\right)$ при $m=1$ принимает вид

$$
V(\alpha)=E_{\alpha}\left(A \alpha^{2}+b\right)
$$

и система бифуркационных уравнений для (37)

$$
A \alpha^{2}+b=0
$$

- это линейная алгебраическая система относительно вектора $\alpha^{2}$.

Предположим, что вектор $\alpha^{*}$ удовлетворяет системе (40), обращая в (39) $V(\alpha)$ в нуль. Тогда в формуле $(19) V_{i}^{(j)}\left(\alpha^{*}\right)=2 a_{i j} \alpha_{i}^{*} \alpha_{j}^{*}$, откуда

$$
V_{L}\left(\alpha^{*}\right)=\left\{V_{i}^{(j)}\left(\alpha^{*}\right)\right\}_{i, j=1}^{d}=2 E_{\alpha^{*}} A E_{\alpha^{*}}
$$

Обозначая через $A_{j}^{b}, j=1, \ldots, d$, матрицу $A$, в которой столбец с номером $j$ заменен на вектор $-b$, наложим на коэффициенты системы (37), входящие в формулы (38), следуюшие ограничения:

$$
b \neq 0, \quad \frac{\operatorname{det} A_{j}^{b}}{\operatorname{det} A}>0, \quad j=1, \ldots, d .
$$

Положим

$$
\alpha^{*}=\left(\left(\frac{\operatorname{det} A_{1}^{b}}{\operatorname{det} A}\right)^{1 / 2}, \ldots,\left(\frac{\operatorname{det} A_{d}^{b}}{\operatorname{det} A}\right)^{1 / 2}\right)
$$

Тогда $\alpha^{*}$ - единственное положительное решение бифуркационной системы (40) и из теоремы 1 вытекает следующее утверждение.

ТЕОрема 2. Пусть для системы (37) с $d \geqslant 2$ выполняется условие (42), вектор $\alpha^{*}$, определенный в (43), удовлетворяет условию (13) и матрича $E_{\alpha^{*}} A E_{\alpha^{*}}$ из (41) некритическая. Тогда при любом достаточно малом $\varepsilon>0$ система (37) имеет $d$-мерный инвариантный тор с частотами движений на нем близкими $к$ $\varepsilon^{1 / 2} \alpha_{1}^{*} \omega^{-1}, \ldots, \varepsilon^{1 / 2} \alpha_{d}^{*} \omega^{-1}$. 
11. Второй частный случай. Система (44). Предположим теперь, что система (37) не зависит от малого положительного параметра $\varepsilon$ и в ней $d=2$. Последнеепредположение не существенно и делается для большей наглядности результатов. Тем самым в (37) $X_{\iota}^{[k]}=X_{\iota k}^{[k]}, \iota=1,2,3,4$, т.е. $X_{\iota k}^{[k-2 \nu]}=0$ при $1 \leqslant \nu \leqslant k / 2$.

Итак, пусть четырехмерная система (37) имеет вид

$$
\begin{array}{ll}
\dot{x}_{1}=x_{3}+X_{1}^{[3]}(x)+X_{1}^{*[4]}(x), & \dot{x}_{3}=-x_{1}^{3}+X_{3}^{[4]}(x)+X_{3}^{*[5]}(x), \\
\dot{x}_{2}=x_{4}+X_{2}^{[3]}(x)+X_{2}^{*[4]}(x), & \dot{x}_{4}=-x_{2}^{3}+X_{4}^{[4]}(x)+X_{4}^{*[5]}(x),
\end{array}
$$

где $X_{i}^{*[4]}(x)=X_{i}^{[4]}(x)+X_{i}^{*[5]}(x), X_{i}^{*[5]}\left(\delta x_{1}, \delta x_{2}, \delta^{2} x_{3}, \delta^{2} x_{4}\right)=\delta^{5} \widehat{X}_{i}^{*[5]}(x, \delta)$, функции $\widehat{X}_{i}^{*[5]}(x, \delta)$ непрерьвны по совокупности аргументов и непрерывно дифференцируемы по $x$ при $\|x\|<x_{0}, 0 \leqslant \delta<\delta_{0}, i=1,2$. И аналогично устроены функции $X_{i+2}^{*[5]}(x)$.

Тогда для системы (44) определенньй в (38) вектор $b=0$, в формуле (39) функция $V(\alpha)=E_{\alpha} A \alpha^{2}$, а бифуркационная система (40) - это линейная однородная система $A \alpha^{2}=0$ или в координатной форме

$$
a_{11} \alpha_{1}^{2}+a_{12} \alpha_{2}^{2}=0, \quad a_{21} \alpha_{1}^{2}+a_{22} \alpha_{2}^{2}=0,
$$

где $a_{11}=\left(3 X_{1}^{(3,0,0,0)}+X_{3}^{(2,0,1,0)}\right) v / 5, a_{22}=\left(3 X_{2}^{(0,3,0,0)}+X_{4}^{(0,2,0,1)}\right) v / 5, a_{12}=$ $\left(X_{1}^{(1,2,0,0)}+X_{3}^{(0,2,1,0)}\right) v / 3, a_{21}=\left(X_{2}^{(2,1,0,0)}+X_{4}^{(2,0,0,1)}\right) v / 3$.

Система (45) разрешима и имеет однопараметрическое семейство решений, если существует такое $\beta>0$, что

$$
-\frac{a_{11}}{a_{12}}=-\frac{a_{21}}{a_{22}}=\beta^{2}>0 .
$$

Пусть условие (46) вьполняется. Положим

$$
\alpha_{c}^{*}=(c, \beta c)=c\left(1,\left(-\frac{a_{11}}{a_{12}}\right)^{1 / 2}\right)=c\left(1,\left(-\frac{a_{21}}{a_{22}}\right)^{1 / 2}\right), \quad c>0 .
$$

Тогда при любом значении $c$ вектор $\alpha_{c}^{*}$ будет являться положительным решением бифуркационной системы (45).

Указанный вектор $\alpha_{c}^{*}$ удовлетворяет условию (13), если

$$
\exists \gamma>0, \quad \tau \geqslant 2: \quad \forall q_{1}, q_{2} \text { - цельх, } \quad q_{1}^{2}+q_{2}^{2} \neq 0 \Rightarrow\left|q_{1}+\beta q_{2}\right|>\gamma\left(\left|q_{1}\right|+\left|q_{2}\right|\right)^{-\tau} .
$$

Действительно, условие (48) непосредственно вьполняется для $\alpha_{c}^{*} c c=1$, а при других $c$ в (48) $\gamma$ надо заменить на $\gamma c^{-1}$.

Далее, в формуле (41) с учетом (46) и (47) матрица $V_{L}\left(\alpha_{c}^{*}\right)$ равна

$$
2\left(\begin{array}{cc}
a_{11} \alpha_{c 1}^{* 2} & a_{12} \alpha_{c 1}^{*} \alpha_{c 2}^{*} \\
a_{21} \alpha_{c 1}^{*} \alpha_{c 2}^{*} & a_{22} \alpha_{c 2}^{* 2}
\end{array}\right)=2 c^{2}\left(\begin{array}{cc}
a_{11} & a_{12}\left(-a_{11} / a_{12}\right)^{1 / 2} \\
a_{21}\left(-a_{11} / a_{12}\right)^{1 / 2} & a_{22}\left(-a_{21} / a_{22}\right)
\end{array}\right) ;
$$

очевидно, при этом элемент $V_{1}^{(2)}=2 c^{2}\left(-a_{11} a_{12}\right)^{1 / 2} \operatorname{sign} a_{12}$. Следовательно, собственные числа $\lambda_{1}$ и $\lambda_{2}$ матрицы $V_{L}\left(\alpha_{c}^{*}\right)$ удовлетворяют уравнению $\lambda^{2}-\left(a_{11}-a_{21}\right) \lambda+$ 
$a_{21}\left|a_{11}\right| \operatorname{sign} a_{12}=0$, откуда $\lambda_{1}=a_{11}, \lambda_{2}=-a_{21}$, а значит, $V_{L}\left(\alpha_{c}^{*}\right)-$ некритическая матрица при любом $c>0$.

Таким образом, для вьполнения условий (18) теоремы 1 применительно к системе (44) для коэффициентов, входящих в бифуркационную систему (45), достаточно потребовать вьполнения условий (46) и (48) при любом векторе $\alpha^{*}$ из (47). Фактически это означает, что коэффициенты однородных полиномов $X_{i}^{[3]}, X_{i+2}^{[4]}$ системы (44), входящие в в систему (45), должны удовлетворять условию, вытекающему из (46):

существует такое $\beta>0$, удовлетворяющее (48), что

$$
\begin{aligned}
3\left(3 X_{1}^{(3,0,0,0)}+X_{3}^{(2,0,1,0)}\right) & =-5 \beta^{2}\left(X_{1}^{(1,2,0,0)}+X_{3}^{(0,2,1,0)}\right), \\
5\left(X_{2}^{(2,1,0,0)}+X_{4}^{(2,0,0,1)}\right) & =-3 \beta^{2}\left(3 X_{2}^{(0,3,0,0)}+X_{4}^{(0,2,0,1)}\right) .
\end{aligned}
$$

Остается разобраться в зависимости между $c$ и $\varepsilon$. Для этого надо последовательно применить к системе (44) замены, описанные в п.п. 2-4.

Пусть в (6) функции $R^{*[3]}, \Phi^{*[2]}$ непрерьвно дифференцируемы по $r_{i}$ и вешественно аналитичны по $\varphi_{i}$ при $\left|r_{i}\right|<r_{0}$. Тогда согласно (47) для всякого $c>0$ в замене (7) с $\alpha=\alpha_{c}^{*}$ должно вьполняться неравенство $\varepsilon<\varepsilon_{c}^{\beta}=r_{0}^{2} /\left(2 c \beta^{0}\right)^{2}, \beta^{0}=\max \{1, \beta\}$. Кроме того, лемма Хейла может быть применена к системе $(25)$, если $\varepsilon<\varepsilon_{c}^{0}$. Возьмем $\varepsilon_{c}=\min \left\{\varepsilon_{c}^{\beta}, \varepsilon_{c}^{0}\right\}$.

ТЕОрема 3. Любая система (44), удовлетворяющая условию (49), при всяком $c>0 u 0<\varepsilon<\varepsilon_{c}$ имеет однопараметрическое семейство двумерных инвариантных торов вида

$$
\begin{aligned}
& x_{1}=\varepsilon^{1 / 2} c \operatorname{Cs} \psi_{1}+\varepsilon\left(\left(\tilde{f}_{1}(\psi)-\bar{g}_{1}\right) \operatorname{Cs} \psi_{1}-c \tilde{\theta}_{1}(\psi) \operatorname{Sn} \psi_{1}\right)+O\left(\varepsilon^{3 / 2}\right), \\
& x_{2}=\varepsilon^{1 / 2} c \beta \operatorname{Cs} \psi_{2}+\varepsilon\left(\left(\tilde{f}_{2}(\psi)-\bar{g}_{2}\right) \operatorname{Cs} \psi_{2}-c \beta \tilde{\theta}_{2}(\psi) \operatorname{Sn} \psi_{2}\right)+O\left(\varepsilon^{3 / 2}\right), \\
& x_{3}=-\varepsilon c^{2} \operatorname{Sn} \psi_{1}-\varepsilon^{3 / 2}\left(2 c\left(\tilde{f}_{1}(\psi)-\bar{g}_{1}\right) \operatorname{Sn} \psi_{1}+c^{2} \tilde{\theta}_{1}(\psi) \operatorname{Cs}^{3} \psi_{1}\right)+O\left(\varepsilon^{2}\right), \\
& x_{4}=-\varepsilon c^{2} \beta^{2} \operatorname{Sn} \psi_{2}-\varepsilon^{3 / 2}\left(2 c \beta\left(\tilde{f}_{2}(\psi)-\bar{g}_{2}\right) \operatorname{Sn} \psi_{2}+c^{2} \beta^{2} \tilde{\theta}_{2}(\psi) \operatorname{Cs}^{3} \psi_{2}\right)+O\left(\varepsilon^{2}\right),
\end{aligned}
$$

с частотами движений на них близкими $к \varepsilon^{1 / 2} c \omega^{-1}, \varepsilon^{1 / 2} c \beta \omega^{-1}$.

Теорема 3 является очевидным следствием теоремы 1 , а формула для семейства инвариантных торов системы (44) вытекает из формулы (36).

12. Заключение. Отметим, что бифуркация положения равновесия системы (1) при $n=1$ исследована в работе [1]. А в работе [4] также при $n=1$ конструктивно построены классы вещественных систем, в том числе полиномиальных, в которых при всех малых значениях параметра происходит бифуркация рождения инвариантного тора размерности на единицу меньшей, чем размерность системы, совпадающей с произвольной степенью числа два.

Кроме того, система (1), в которой $d=2$ и четвертое уравнение имеет вид $\dot{x}_{4}=$ $-x_{2}^{n+1}+X_{4}^{2 n+2}(x, \varepsilon)+X_{4}^{* 2 n+3}(x, \varepsilon)$, при $n=1$ изучалась в работах [5]-[7], а при $n \geqslant 2$ - в работе [8]. Для системы типа (48) это означает, что два слабосвязанных осциллятора имеют восстанавливающие силы ближайших, но различных нечетных порядков $2 n-1$ и $2 n+1$.

Комбинирование используемых в перечисленных работах результатов и методов в принципе позволяет исследовать любую систему с нулевым приближением $\left(\lambda_{1} x_{d+1}, \ldots\right.$, $\left.\lambda_{d} x_{2 d},-\lambda_{1} x_{1}^{2 n_{1}-1}, \ldots,-\lambda_{d} x_{d}^{2 n_{d}-1}\right)$. 


\section{СПИСОК ЦИТИРОВАННОЙ ЛИТЕРАТУРЫ}

[1] Бибиков Ю. Н. Многочастотные нелинейные колебания и их бифуркации. Л.: Изд-во Ленинградского ун-та, 1991.

[2] Ляпунов А. М. Исследование одного из особенных случаев задачи об устойчивости движения // Собр. соч. Т. 2. М., Л.: Изд-во АН СССР, 1956. С. 272-331.

[3] Hale J. K. Integral manifolds of perturbed differential systems // Ann. of Math. 1961. V. 73. № 3. P. 496-531.

[4] Басов В. В. Бифуркация инвариантного тора коразмерности единица // Матем. заметки. 2001. T. 69. № 1. С. $3-17$.

[5] Басов В. В. Об устойчивости положения равновесия в критическом случае двух чисто-мнимых и двух нулевых корней характеристического уравнения // Дифференц. уравнения. 1999. Т. 35. № 10. С. 1313-1318.

[6] Басов В. В., Бибиков Ю. Н. Бифуркация положения равновесия системы дифференциальных уравнений в критическом случае двух чисто мнимых и двух нулевых корней характеристического уравнения, I // Дифференц. уравнения. 2000. Т. 36. № 1. С. 26-32.

[7] Басов В. В. Бифуркация положения равновесия системы дифференциальных уравнений в критическом случае двух чисто мнимых и двух нулевых корней характеристического уравнения, II // Дифференц. уравнения. 2001. Т. 37. № 4. С. 435-438.

[8] Басов В. В. Бифуркация положения равновесия системы дифференциальных уравнений в критическом случае двух пар нулевых корней характеристического уравнения // Тр. МИPAH. 2002. T. 236. C. 45-60.

Санкт-Петербургский государственный университет

Поступило

E-mail : basov@VB2674.spb.edu

24.12 .2002

Исправленный вариант

11.06.2003 